have arrived at a similar viewpoint in recent years. Personally, I have yet to be persuaded that ethology can dispense with objectivism, or that there are methods to study subjective phenomena objectively: but the field is an important and open one, and de Waal's view of it certainly deserves a careful hearing.

De Waal writes in everyday language, evidently with the general reader as well as the specialist in mind, but he manages to avoid many of the pitfalls of popular science writing. There are some lapses, however, both on particular subjects (e.g. social anthropology) and in coat-trailing to keep the reader's attention. A good impression of an ethologist's approach and evidence as well as his conclusions is given by such things as the inclusion of a few clear diagrams: I wish he had been bolder and supplied more of these, as well as cited evidence more specifically. Although I take with a pinch of salt the claim that "whole passages of Machiavelli seem to be directly applicable to chimpanzee behaviour", there is no doubting the fascinating complexity of chimpanzee social life: and de Waal's study of it is important both as empirical research and as a discussion of ideas.

Robert Attenborough is a Lecturer in Biological Anthropology in the Department of Prehistory and Anthropology, Australian National University, Canberra.

\section{Why are there so few species of animals?}

\section{E. C. Pielou}

Resource Competition and Community Structure.

By David Tilman.

Princeton University Press: 1982. Pp.296. Hbk \$27.50, £23.70; pbk \$9.95, £8.70.

DAVID TILMAN has written an outstandingly good book. In it he develops a new, extremely well-argued approach to the perennially fascinating question: why have so many terrestrial plant species evolved (there are about 0.3 million) and how do they manage to coexist, in wellmingled species-rich communities, even though all have the same few needs of light, water, carbon dioxide and a few mineral nutrients? The profusion of plant species seems (until you read Tilman) far harder to account for than the profusion of animal species. Animals, even those at the bottom of the trophic pyramid, have available to them all the different parts of all plants, in round numbers about one million distinct resources. Tilman estimates that the number of species per resource is about $10^{4}$ times as great in plants as in animals.

He shows how this high level of plant species diversity can be explained if one postulates that the competitive success of a species depends on the relative rather than the absolute rates of supply of different resources. Spatial and temporal changes in resource ratios play an important role too. A hitherto unpublished figure (Fig. 35) gives isopleth maps of total nitrogen, extractable magnesium, and their ratio, in a $12 \times 12 \mathrm{~m}$ plot of soil. The intricacy of the small-scale pattern is noteworthy and might have been even more conspicuous if a three-dimensional study had been made, taking depth into account.

Tilman shows how niche diversification is possible among a collection of species even when they are competing for only two essential nutrients. His theory also explains very satisfactorily why species richness is nearly always greatest in habitats that are fairly poor in resources. However, the argument that temperate soils are often richer in nutrients than tropical soils, and that here at last is an explanation for the famous latitudinal diversity gradient, is unfortunate. As P. W. Richards, in his contribution to Speciation in Tropical Environments (Academic, 1969) has remarked, plant species diversity does not exhibit a clear latitudinal gradient (except in rain forest); the phenomenon is chiefly confined to animals.

Tilman develops his theory of competition for resources with a liberal use of isocline graphs. When the postulates are put on paper in this form, not only are the consequences that follow immediately visible but in addition the logical unfolding of his theory becomes a pleasure to follow.

The theoretical development is accompanied by careful and detailed discussion of a wide variety of actual communities ranging from cultures of diatoms and bluegreen algae to forests. This brings me to what seems the only real weakness in the whole theory: no allowance is made for delays in the responses of a plant to changes in the rate of supply of its resources. Obviously blue-green algae respond faster than trees. The complete neglect of time lags is the one serious fault in the whole edifice; because the theory is so satisfying, I hope Tilman will be able to show that it is robust enough to survive the incorporation of this additional factor.

In a fascinating concluding chapter, Tilman sums up his answer to the question posed at the beginning of this review, which boils down to: why are there so few animal species? In a nutshell, his answer is that terrestrial plant species each require essential (non-substitutable) nutrients in specific ratios. Mobile animals, by contrast, tend to be able to substitute one resource for another if shortages occur. The contrast is thus between sessile specialists and mobile generalists, and the mystery disappears.

E. C. Pielou is Alberta Oil Sands Environmental Research Professor in the Department of Biology at the University of Lethbridge, Alberta.

\section{Going to the pictures}

\author{
P.W. Hawkes
}

Digital Picture Processing, 2nd Edn.

By Azriel Rosenfeld and Avinash C. Kak. Academic: 1982. Vol.1 pp.434, \$35, £23.20; Vol.2 pp.343, \$29, £19.20.

THE FIRST edition of Digital Picture Processing appeared in 1976 and rapidly became the standard textbook of the subject, rivalled but not ousted by the few other good texts that appeared not long after. The subject is in almost explosive expansion however - at least two new journals and a "Progress" have recently been devoted to it - and the 1976 edition is no longer adequate as a course text.

The authors have added an immense amount of new material (Vol.1 alone of the new edition is as long as the 1976 book) and revised many passages that had become inadequate, though not to the point of desuetude. Specifically, whole chapters have been added on reconstruction from projections and on matching, most of which are new. The chapter on image compression, which badly needed bringing up to date, has been extensively rewritten so that the fast Karhunen-Loève transform and the cosine transform are now covered properly. It has also been possible to include block truncation compression.

The chapter on restoration has also been expanded, but is disappointing in one respect. The account of recursive methods in the first edition was by far the most readable I have seen of this difficult topic, and there have been many important developments since 1976; few of these have been incorporated though the section has been somewhat extended.

The other major theme of the work is picture analysis, which includes but transcends pattern recognition, and fills the second volume. The chapter on segmentation now includes a section on iterative relaxation methods for probabilistic segmentation, which will heip the reader to understand fuzzy papers (in the technical sense), as well as new material on such topics as Hough transforms and edge detection. The final two chapters on representations and image description, notoriously difficult subjects for the beginner owing to the seemingly haphazard connections between different aspects, have been reorganized in a respectable attempt to impose a pattern on patterns.

I doubt whether it will be difficult to persuade users of the first edition to acquire the second. Those who are only just entering this fascinating but complex field will be very foolish not to equip themselves with this excellent, well-written and authoritative guide.

P.W. Hawkes is Maitre de Recherches at the Laboratoire d'Optique Electronique du CNRS, Toulouse. 